\title{
Distinguishing Refracted Features using Light Field Cameras with Application to Structure from Motion
}

\author{
Dorian Tsai ${ }^{1}$, Donald G. Dansereau ${ }^{2}$, Thierry Peynot ${ }^{1}$ and Peter Corke ${ }^{1}$
}

\begin{abstract}
Robots must reliably interact with refractive objects in many applications; however, refractive objects can cause many robotic vision algorithms to become unreliable or even fail, particularly feature-based matching applications, such as structure-from-motion. We propose a method to distinguish between refracted and Lambertian image features using a light field camera. Specifically, we propose to use textural cross-correlation to characterise apparent feature motion in a single light field, and compare this motion to its Lambertian equivalent based on 4D light field geometry. Our refracted feature distinguisher has a $34.3 \%$ higher rate of detection compared to state-of-the-art for light fields captured with large baselines relative to the refractive object. Our method also applies to light field cameras with much smaller baselines than previously considered, yielding up to 2 times better detection for 2D-refractive objects, such as a sphere, and up to 8 times better for 1D-refractive objects, such as a cylinder. For structure from motion, we demonstrate that rejecting refracted features using our distinguisher yields up to $\mathbf{4 2 . 4 \%}$ lower reprojection error, and lower failure rate when the robot is approaching refractive objects. Our method lead to more robust robot vision in the presence of refractive objects.
\end{abstract}

\section{INTRODUCTION}

Robots for the real world will inevitably interact with refractive objects. Robots must contend with wine glasses and clear water bottles in domestic applications [1]; glass and clear plastic packaging for quality assessment and packing in manufacturing [2]; as well as water and ice for outdoor operations [3]. All of these applications typically require either object structure and/or robot motion to automate. Structure from motion (SfM) is a technique to recover both scene structure and camera pose from $2 \mathrm{D}$ images, and is widely applicable to many systems in computer and robotic vision [4], [5]. Many of these systems assume the scene is Lambertian, in that a 3D point's appearance in an image does not change significantly with viewpoint. However, nonLambertian effects, including specular reflections, occlusions, and refraction, violate this assumption. They pose a major problem for modern robotic vision systems because their appearance depends on the camera's viewing pose and the visual texture of the object's background.

Image features are distinct points of interest in the scene that can be repeatedly and reliably identified from different

This research was partly supported by the Australian Research Council (ARC) Centre of Excellence for Robotic Vision (CE140100016).

${ }^{1}$ D. Tsai, T. Peynot and P. Corke are with the Australian Centre for Robotic Vision (ACRV), Queensland University of Technology (QUT), Brisbane, Australia \{dy.tsai, t.peynot, peter.corke\} @qut.edu.au

${ }^{2}$ D. Dansereau is with the Stanford Computational Imaging Lab, Stanford University, CA, USA. donald.dansereaulgmail.com

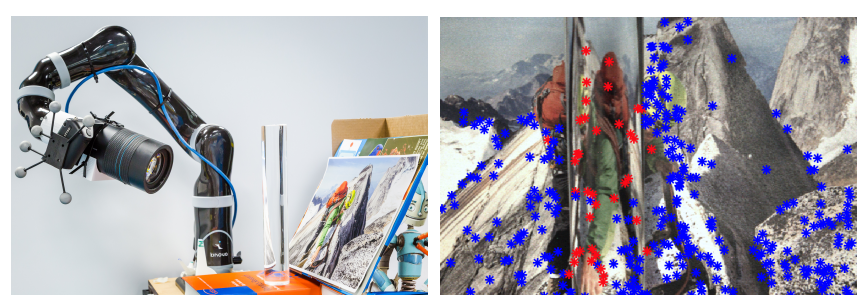

Fig. 1. (left) A light field camera mounted on a robot arm was used to distinguish refractive objects in a scene. (right) SIFT features that were distinguished as Lambertian (blue) and refracted (red), indicating the refractive cylinder in the middle of the scene.

viewpoints, and have been used in SfM, but also many other robotic vision algorithms, such as object recognition, image segmentation, visual servoing, visual odometry, and simultaneous localization and mapping (SLAM). In SfM, features are often used for image registration. When reconstructing a scene containing a refractive object, such as Fig. 1, image features occluded by the object appear to move differently from the rest of the scene. They can cause inconsistencies, errors, and even failures for modern robotic vision systems.

Light field (LF) cameras offer a potential solution to the problem of refractive objects. LF cameras simultaneously capture multiple images of the same scene from different viewpoints in a regular and dense sampling. The LF could allow robots to more reliably and efficiently capture the behaviour of refractive objects in a single shot by exploiting the known geometry of the multiple views. We take 2D image features from the central view of the LF, and determine which of these exhibit refractive behaviour in the 4D LF, which we refer to as a refracted feature, and use this as a method of distinguishing good features for SfM.

Our main contributions are the following.

- We extend previous work to develop a light field feature distinguisher for refractive objects. In particular, we detect the differences between the apparent motion of non-Lambertian and Lambertian features in the 4D light field to distinguish refractive objects more reliably than previous work.

- We propose a novel approach to describe the apparent motion of a feature observed within the 4D light field based on textural cross-correlation.

- We extend refracted feature distinguishing capabilities to lenslet-based LF cameras that are limited to much smaller baselines by considering non-uniform, nonLambertian apparent motion in the light field. All light fields captured for these experiments will be available online at https://tinyurl.com/LFRefractive. 
Our method outperforms the state of the art in terms of detecting refracted features, including small-baseline LF cameras for the first time. We also show that rejecting refracted features before the SfM pipeline can yield lower reprojection error in the presence of refractive objects, provided there are a sufficient number of Lambertian features remaining.

The main limitation of our method is that it requires background visual texture distorted by the refractive object. Our method's effectiveness depends on the extent to which the appearance of the object is warped in the light field. This depends on the geometry and refractive indices of the object involved.

The remainder of this paper is organized as follows. We describe the related work, provide background on LF geometry, and explain our method for distinguishing refracted features. Then we show our experimental results for extraction of a feature's apparent motion, detection with different camera baselines and object types, and validation in the context of monocular SfM. Finally, we conclude the paper and explore future work.

\section{RELATED WORK}

A variety of strategies for detecting, and even reconstructing refractive objects using vision have been investigated [2]. However, many of these methods require known light sources with bulky configurations that are impractical for mobile robot applications. Multiple monocular images have been used to recover refractive object shape and pose [6]; however, image features were manually tagged throughout camera motion, emphasizing the difficulty of automatically identifying and tracking refracted features due to the severe magnification of the background, and changes in image intensities when passing through the object.

LFs have recently been used to explore refractive objects. Wanner et al. considered planar refractive surfaces and reconstructed different depth layers that accounted for both refraction through a thin sheet of glass, and the reflection caused by its glossy surface [7]. However, this work was limited to thin planar surfaces and single reflections. Which depth layer was Lambertian, reflective or refractive was not distinguished, and refractive objects that caused significant distortion were not handled. Although our work does not determine the dense structure of the refractive object, our approach can distinguish features from objects that significantly distort the LF.

For refractive object recognition, Maeno et al. proposed a light field distortion feature (LFD), which models an object's refraction pattern as image distortion based on differences in the corresponding image points between the multiple views of the LF, captured by a large-baseline (relative to the refractive object) LF camera array [8]. However, the authors observed significantly poor recognition performance due to specular reflections, as well as changes in camera pose.

$\mathrm{Xu}$ et al. used the LFD as a basis for refractive object image segmentation [9]. Corresponding image features from all views in the LF were fitted to the single normal of a 4D hyperplane in the least-squares sense using singular value decomposition (SVD). The smallest singular value was taken as a measure of error to the hyperplane of best fit, for which a threshold was applied to distinguish refracted features. However, as we will show in this paper, a 3D point cannot be described by a single hyperplane in 4D. Instead, it manifests as a plane in 4D that has two orthogonal normal vectors. Our approach builds on Xu's method, and solves for both normals to find the plane of best fit in 4D; thus allowing us to distinguish more types of refractive objects with a higher rate of detection.

Furthermore, a key difficulty in feature-based approaches in the LF is obtaining the corresponding feature locations between multiple views. Both Maeno and $\mathrm{Xu}$ used optical flow between two views for correspondence, which does not exploit the unique geometry of the LF. We propose a novel textural cross-correlation method to correspond features in the LF by describing their apparent motion in the LF, which we refer to as feature curves. This method directly exploits LF geometry and provides insight on the 4D nature of features in the LF.

Our interest in LF cameras stems from robot applications that often have mass, power and size constraints. Thus, we are interested in employing compact lenslet-based LF cameras to deal with refractive objects. However, most previous works have utilized gantries [7], or large camera arrays [8], [9]; their results do not transfer to LF cameras with much smaller baselines, where refractive behaviour is less apparent, as we show later. We then demonstrate the performance of our method over two different LF camera baselines, and two different LF camera architectures. We demonstrate refracted feature identification with a lenslet-based LF camera, which to the best of our knowledge, has not been done before.

For LF cameras, LF-specific features have been investigated. SIFT features augmented with "slope", an LF-based property related to depth, were proposed by the authors for visual servoing using a LF camera [10]; however, refraction was not considered in prior work. Tosic developed a scaleinvariant, single pixel, edge detector by finding local extrema in a combined scale, depth, and image space [11]. However, these LF features did not differentiate between Lambertian and refracted features, nor were they designed for reliable matching between LFs captured from different viewpoints. In this paper, we detect unique keypoints that reject refracted content and work well for SfM.

Recent work by Teixeira found SIFT features in all views of the LF and projected them into their corresponding epipolar plane images (EPI). These projections were filtered and grouped onto straight lines in their respective EPIs, then counted. Features with higher counts were observed in more views, and thus considered more reliable [12]. However, their approach did not consider any non-linear feature behaviour, while our proposed method aims at detecting these nonLambertian features, and is focused on characterising them. This could be useful for many feature-based algorithms, including recognition, segmentation, visual servoing, simultaneous localization and mapping, visual odometry, and SfM. 
We are interested in exploring the impact of our refracted feature distinguisher in a SfM framework. While there has been significant development in SfM in recent years [5], Johannsen was the first to consider LFs in the SfM framework [13]. As a first step, our work does not yet explore LFbased SfM. Instead, we investigate SfM's performance with respect to refracted features, which has not yet been fully explored. We show that rejecting refracted features reduces reprojection error and failure rate near refractive objects.

\section{LIGHT FIELD BACKGROUND}

We parameterize the LF using the relative two-plane parameterization (2PP) [10]. A ray with coordinates $\phi=[s, t, u, v]^{T}$, where ${ }^{T}$ represents the vector transpose, is described by two points of intersection with two parallel reference planes; an $s, t$ plane conventionally closest to the camera, and a $u, v$ plane conventionally closer to the scene, separated by arbitrary distance $D$.

For a Lambertian point in space $\boldsymbol{P}=\left[P_{x}, P_{y}, P_{z}\right]^{T} \in \mathbb{R}^{3}$, the rays follow a linear relationship

$$
\left[\begin{array}{l}
u \\
v
\end{array}\right]=\left(\frac{D}{P_{z}}\right)\left[\begin{array}{l}
P_{x}-s \\
P_{y}-t
\end{array}\right]
$$

where each of these equations describes a hyperplane in 4D. In this paper, a hyperplane is defined as a vector subspace that has 1 dimension less than the space it is contained within [14]. Thus a hyperplane in 4D is a 3-dimensional manifold, and can be described by a single equation

$$
n_{1} s+n_{2} t+n_{3} u+n_{4} v+n_{5}=0
$$

where $\boldsymbol{n}=\left[n_{1}, n_{2}, n_{3}, n_{4}\right]^{T}$ is the normal of the hyperplane. Similarly, a plane is defined as a 2-dimensional manifold; in other words, it can be described by two linearly independent vectors. Therefore, a plane in $4 \mathrm{D}$ can be defined by the intersection of two hyperplanes and (1) can be re-written in the form,

$$
\underbrace{\left[\begin{array}{cccc}
\frac{D}{P_{z}} & 0 & 1 & 0 \\
0 & \frac{D}{P_{z}} & 0 & 1
\end{array}\right]}_{\boldsymbol{m}}\left[\begin{array}{c}
s \\
t \\
u \\
v
\end{array}\right]=\left[\begin{array}{c}
\frac{D P_{x}}{P} \\
\frac{D \tilde{P}_{y}}{P_{z}}
\end{array}\right],
$$

where $\boldsymbol{m}$ contains the two orthogonal normals to the plane. Therefore, a Lambertian point in 3D manifests itself as a plane in $4 \mathrm{D}$, which is characterized by two linearlyindependent normal vectors that each define a hyperplane in 4D. In the literature, this relationship is sometimes referred to as the point-plane correspondence.

Light field slope $w$ relates the rate of change of image plane coordinates, with respect to viewpoint position, for all rays emanating from a point in the scene. In the literature, slope is sometimes referred to as "orientation" [7], and other works compute slope as an angle [11]. The slope comes directly from (1) as

$$
w=-\frac{D}{P_{z}},
$$

and is clearly related to depth. Importantly, slope is uniform; it is identical for the $s, u$ and $t, v$ planes for a Lambertian point.

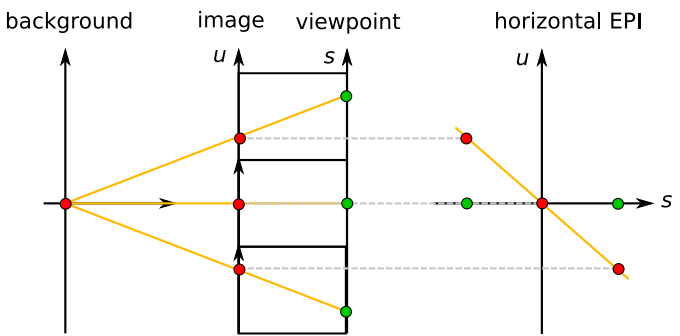

(a)

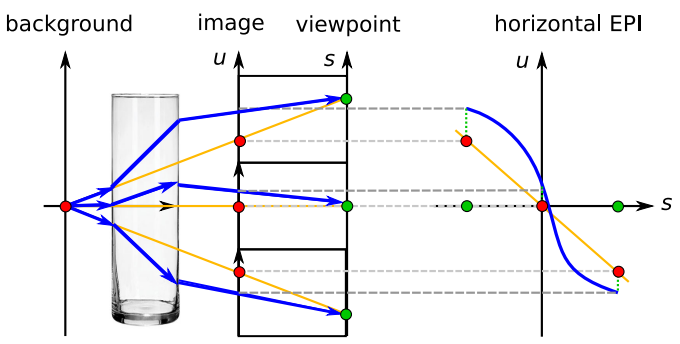

(b)

Fig. 2. (a) Projection of the linear behaviour of a Lambertian feature, and (b) the non-linear behaviour of a refracted feature with respect to linear motion along the viewpoints of a light field.

\section{DISTINGUISHING REFRACTIVE FEATURES}

Epipolar planar images (EPIs) graphically illustrate the apparent motion of a feature across multiple views [15]. If the entire light field $L$ is given as $L(s, t, u, v)$, EPIs represent a $2 \mathrm{D}$ slice of the $4 \mathrm{D}$ LF. A horizontal EPI is given as $L\left(s, t^{*}, u, v^{*}\right)$, and a vertical EPI is denoted as $L\left(s^{*}, t, u^{*}, v\right)$, where ${ }^{*}$ indicates a variable is fixed while others are allowed to vary. The central view of the LF is given as $L\left(s_{0}, t_{0}, u, v\right)$, and is equivalent to what a monocular camera would provide from the same pose. As shown in Fig. 2, features from a Lambertian scene point are linearly distributed with respect to viewpoint, unlike features from highly-distorting refractive objects. We compare this difference in apparent motion between Lambertian and nonLambertian features to distinguish refracted features.

Fig. 3 shows the central view and an example EPI of a crystal ball LF (large baseline) from the New Stanford Light Field Archive [16]. A Lambertian point forms a straight line in the EPI, which represents one of the point's hyperplanes in 4D, as illustrated by the Lambertian scene content in Fig. 3b, i.e. to the top $(v<100)$, and bottom $(v>200)$ of the crystal ball. The relation between slope and depth is also apparent in this EPI.

For a refracted feature, such as those seen in Fig. 3b for $100<v<200$ pix, detection in the LF simplifies to finding features that violate (1) via identifying non-linear feature curves in the EPIs and/or inconsistent slopes between two independent EPI lines, such as the vertical and horizontal EPIs. We note that occlusions and specular reflections also violate (1). Occlusions appear as straight lines, but have intersections in the EPI. Edges of the refractive objects, and objects with low distortion also appear Lambertian. Specular reflections appear as a superposition of lines in the EPI. We will address these issues in future work. For now, we discuss 


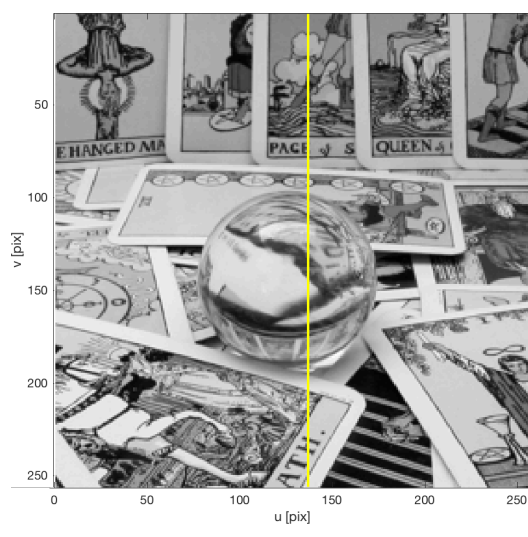

(a)

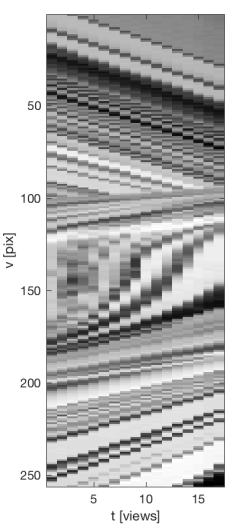

(b)
Fig. 3. (a) In the crystal ball LF [16], a vertical EPI is sampled from a column of pixels (yellow), where (b) nonlinear refracted feature motion caused by the crystal ball are apparent in the middle of this vertical EPI. Straight lines from $v<100$, and $v>200$ relate to Lambertian features.

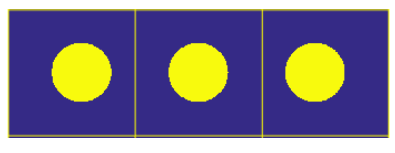

(a)

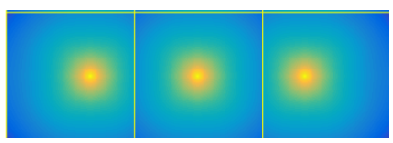

(c)

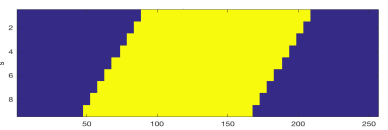

(b)

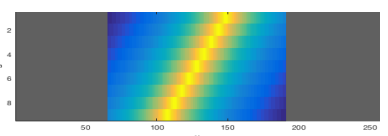

(d)
Fig. 4. (a) Simulated horizontal views of a yellow circle. (b) Corresponding EPI taken along the middle of the views. (c) The cross-correlation response for the corresponding views. (d) The resultant correlation EPI, created by stacking the cross-correlation response from adjacent views. The peaks of this correlation EPI correspond to the desired feature curve.

how we extracted these 4D feature curves, and then describe how we identify refracted features.

\section{A. Extracting Feature Curves}

For a given feature from the central view at coordinates $\left(u_{0}, v_{0}\right)$, we must determine the feature correspondences $\left(u^{\prime}, v^{\prime}\right)$ from the other views, which is equivalent to finding the feature's apparent motion, or curves in the LF. In this paper, we start by detecting SIFT features [17] in the central view, although the proposed method is agnostic to feature type.

Next, we select a template surrounding the feature which is $k$-times the feature's scale. We determined $k=5$ to yield the most consistent results. 2D Gaussian-weighted normalized cross-correlation (WNCC) is used across views to yield images, such as Fig. 4c. To reduce computation, we only apply WNCC along a subset of the central row and column relative to the central view of the LF.

For Lambertian features, we plot the feature's correlation response with respect to the views to yield a correlation EPI. Illustrated in Fig. 4d, the peaks of the correlation EPI correspond to the feature curve from original EPI.

For refracted features, we hypothesize that the correlation response will be sufficiently strong that peak values of the correlation EPI will still correspond to the desired feature curve. As such, we threshold the correlation EPI into a binary mask to reduce the area considered for the feature curve. We then take the peak values of the connected component that contains our feature. We apply several line consistency thresholds to remove incorrectly-detected feature curves due to edge boundaries or peak values corresponding to the wrong features. Thresholds were tailored for the specific application. This textural cross-correlation method allows us to focus on the image structure, as opposed to the image intensities, can be applied to any LF camera, and directly exploits the geometry of the LF.

\section{B. Fitting 4D Planarity to Feature Curves}

Similar to [9], we consider the feature ray $\phi\left(0,0, u_{0}, v_{0}\right)$. The corresponding feature coordinates in other views are $\phi^{\prime}\left(s, t, u^{\prime}, v^{\prime}\right)$. The LFD is then defined as the set of relative differences between $\phi$ and $\phi^{\prime}$ :

$$
L F D(u, v)=\{(s, t, \Delta u, \Delta v) \mid(s, t)=(0,0)\},
$$

where $\Delta u=u^{\prime}-u_{0}$, and $\Delta v=v^{\prime}-v_{0}$ are feature disparities.

As illustrated in Fig. 2a, these disparities are linear with respect to linear camera translation. The disparities from refracted features deviate from this linear relation. Fitting them to (1) yields the plane of best fit in 4D, and the error of this fit provides a measure of whether or not our feature is Lambertian.

This plane in 4D can be estimated from the feature correspondences given by the feature curves $f_{h}\left(s, t^{*}, \Delta u, v^{*}-v_{0}\right)$, and $f_{v}\left(s^{*}, t, u^{*}-u_{0}, \Delta v\right)$ that we extract from the horizontal and vertical EPIs, respectively.

As discussed in Section III, our plane in 4D has two orthogonal normals, $\boldsymbol{n}_{h}$ and $\boldsymbol{n}_{v}$. The 4D plane containing $\phi$ can be given as

$$
\left[\begin{array}{llll}
n_{h, 1} & n_{h, 2} & n_{h, 3} & n_{h, 4} \\
n_{v, 1} & n_{v, 2} & n_{v, 3} & n_{v, 4}
\end{array}\right]\left[\begin{array}{c}
s \\
t \\
\Delta u \\
\Delta v
\end{array}\right]=\left[\begin{array}{l}
0 \\
0
\end{array}\right] .
$$

Note that the constants on the right-hand side of (3) cancel out because we consider the differences relative to $u_{0}$ and $v_{0}$. The positions for $s, t$ can be obtained by calibration [18], although the non-linearity behaviour still holds when working with units of "views" for $s, t$.

We can estimate $\boldsymbol{n}_{h}$ and $\boldsymbol{n}_{v}$ by fitting the $N$ points from $f_{h}$ and $M$ points from $f_{v}$ into the system,

$$
\underbrace{\left[\begin{array}{cccc}
(s, & t^{*}, & \Delta u, & \left.v^{*}-v_{0}\right)_{1} \\
\vdots & \vdots & \vdots & \vdots \\
(s, & t^{*}, & \Delta u, & \left.v^{*}-v_{0}\right)_{N} \\
\left(s^{*},\right. & t, & u^{*}-u_{0}, & \Delta v)_{1} \\
\vdots & \vdots & \vdots & \vdots \\
\left(s^{*},\right. & t, & u^{*}-u_{0}, & \Delta v)_{M}
\end{array}\right]}_{\boldsymbol{A}} \underbrace{\left[\begin{array}{c}
n_{1} \\
n_{2} \\
n_{3} \\
n_{4}
\end{array}\right]}_{\boldsymbol{n}}=\mathbf{0 .}
$$

We then use SVD on $\boldsymbol{A}$ to compute the singular vectors, and corresponding singular values. The 2 smallest singular values, $e_{1}$ and $e_{2}$, correspond to 2 normals $\boldsymbol{n}_{1}$ and $\boldsymbol{n}_{2}$ that 
best satisfy (7) in the least-squares sense. The magnitude of the singular values provides an error measure of the planar fit. Smaller errors imply stronger linearity, while larger errors imply that the feature deviates from the 4D plane.

The norm of $e_{1}$ and $e_{2}$ may be taken as a single measure of planarity; however, doing so masks the case where a refractive object has unequal errors between the two EPIs, such as a 1D refractive object (glass cylinder) that is highly refractive along one direction, but almost Lambertian along the other. Therefore, we reject those features that have large errors in either horizontal or vertical hyperplanes, according to an empirical threshold. This planar consistency, along with the slope consistency measure discussed in the following section, make the proposed method more sensitive to refracted texture than prior work that considers only hyperplanar consistency [9].

While $\mathrm{Xu}$ also applies occlusion handling and a graph segmentation framework to complete their transparent object image segmentation algorithm, simply taking the smallest singular value allows us to directly compare the underlying principles for our feature distinguisher.

\section{Measuring Slope Consistency}

Slope consistency is a measure of how similar the slopes are between the two hyperplanes for a given feature. As seen in (1), these slopes must be equal for Lambertian points. We can compute the slopes for each hyperplane given their normals. For the horizontal hyperplane, we solve for in-plane vector $\boldsymbol{q}=\left[q_{s}, q_{u}\right]^{T}$, by taking the inner product of the two vectors in

$$
\left[\begin{array}{ll}
n_{h, 1} & n_{h, 3} \\
n_{v, 1} & n_{v, 3}
\end{array}\right]\left[\begin{array}{l}
q_{s} \\
q_{u}
\end{array}\right]=\mathbf{o},
$$

where $\boldsymbol{q}$ is constrained to the $s, u$ plane, because we choose the first and third elements of $\boldsymbol{n}_{h}$ and $\boldsymbol{n}_{v}$. This system is solved using SVD, and the minimum singular vector yields $\boldsymbol{q}$. The slope for the horizontal hyperplane, $w_{s u}$ is then

$$
w_{s u}=\frac{q_{s}}{q_{u}} .
$$

The slope for the vertical hyperplane $w_{t v}$ is similarly computed from the second and fourth elements of the normal vectors. Alternatively, one can also find the line of best fit for the $s, u$-values in $f_{h}$, and the $t, v$-values in $f_{v}$. Slope inconsistency $c$ is calculated as the square of differences between slopes. Finally, a threshold for slope inconsistency is applied, which is tuned for the application. Features with very inconsistent slopes and large planar errors are identified as belonging to a highly-distorting refractive object, which we refer to as a refracted feature.

\section{EXPERIMENTAL RESULTS}

First, we present our experimental set-up. Second, we present results of our feature extraction method for Lambertian and refracted features. Third, we apply our methods to LFs captured with different baselines. Fourth, we apply our methods to LFs captured with a lenslet-based LF camera to compare different refractive object types. Finally, we use our method to reject refracted features for monocular SfM in the presence of refractive objects, and investigate the impact of our approach.

\section{A. Experimental Set-up}

We used the Stanford New Light Field Database [16], which provided LFs captured from a Lego gantry with a $17 \times 17$ grid of rectified $1024 \times 1024$-pixel images that were down-sampled to $256 \times 256$ pixels to reduce computation. We focused on two LFs that both captured the same scene of a crystal ball surrounded by textured tarot cards. The first was captured with a large baseline $(16.1 \mathrm{~mm} /$ view over 275 $\mathrm{mm})$, while the second was captured with a smaller baseline ( $3.7 \mathrm{~mm} /$ view over $64 \mathrm{~mm})$. This allowed us to compare the effect of LF camera baseline for refracted features.

Even smaller baselines were considered using a lensletbased LF camera. Also known as a plenoptic camera, these LF cameras are of interest to robotics due to their simultaneous view capture, and typically lower size and mass, compared to LF camera arrays and gantries. In this section, the Lytro Illum was used to capture LFs with $15 \times 15$ views, each $433 \times 625$ pixels. Dansereau's Light Field Toolbox was used to decode the LFs from raw LF imagery to the 2PP [18]. To compensate for the extreme lens distortion of the Illum, we removed the outer views, reducing our LF to $13 \times 13$ views. The LF camera was fixed at $100 \mathrm{~mm}$ zoom. For these optics, the Illum was roughly equivalent to a $1.1 \mathrm{~mm} / \mathrm{view}$ over $16.6 \mathrm{~mm}$ LF camera array.

It is important to remember that our results depend on a number of factors. First, the shape and size of the object dictates how the light is refracted. Higher curvature and thickness yield more warping. Second, viewing distance, and background distance to the object directly affect how much distortion can be observed. The closer either is to the object, the more refraction can be observed. Similarly, a larger camera baseline captures more refraction. When possible, these factors were held constant throughout different experiments.

\section{B. Feature Curve Extraction}

We first considered our textural cross-correlation approach, which worked well for Lambertian features. For such a feature the correlation EPI is shown as a surface in Fig. 5a with the feature from the central view shown as the red dot. The corresponding peaks from the other views are shown as the red line. This line corresponds to the feature curve in the image space, as shown in Fig. 5b. Line consistency thresholds were implemented to ensure consistent feature curves. Although not implemented, we can limit the crosscorrelation space, since we know the features move with some maximum slope, to further reduce computation.

For refracted features, our approach captured the feature curves. For a typical refracted SIFT feature, the correlation EPI is shown in Fig. 5c. We observed that while the correlation function often had a much weaker response compared to the Lambertian case, local maxima were still observed near the original feature's spatial location in the central view. 


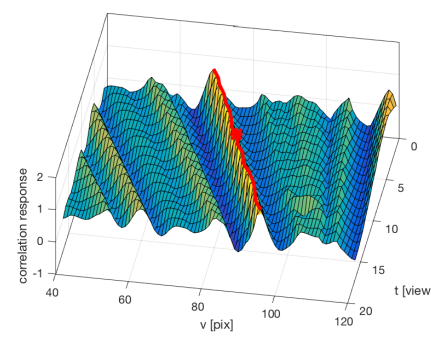

(a)

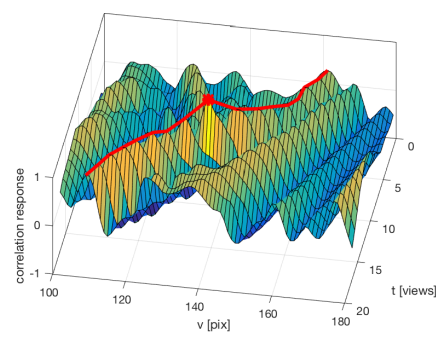

(c)

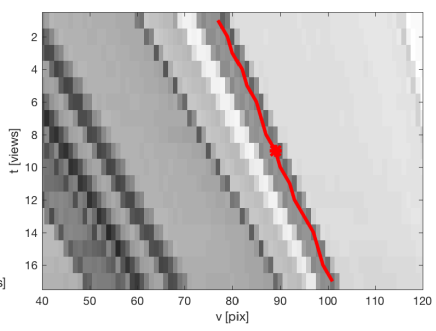

(b)

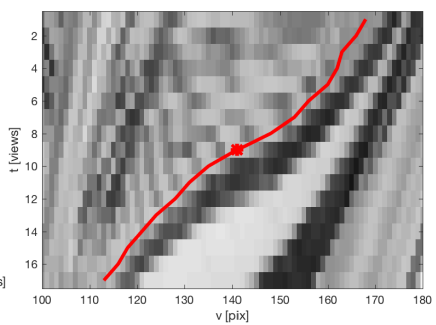

(d)
Fig. 5. (a) The straight Lambertian feature curve (red) from the correlation EPI corresponds to (b) the feature curve in the original EPI. (c) The refracted feature curve (red) in the correlation EPI can still be extracted, despite more complex "terrain", and still matches (d) the curve just above the refracted black curve in the original EPI.

Thus, taking the local maxima of the correlation EPI still yielded the desired feature curve, as shown in Fig. 5d.

Our method relies on thresholds that we have set empirically, which results in limited flexibility to changing environmental conditions. As future work, we are considering implementing ridge-following methods to improve the feature curve extraction. Nonetheless, our textural crosscorrelation method enabled us to extract refracted feature curves without focusing on image intensities in a way that exploited the geometry of the LF.

\section{Refracted Feature Comparison for LF Camera Baseline}

For implementation, the thresholds for planarity and slope consistency were manually applied in order to provide optimal results for both Xu's and our approach, independently. For metrics, the number of refracted feature detections and false positives were counted, and compared. The true positive rate (TPR) was given as the number of refracted features detected in the central view within the image space occupied by the refractive object, over the number of actual refracted features, obtained via a mask identified by the author for the refractive object in the central view. The false positive rate (FPR) was calculated as the number of features falsely identified as refracted over the number of features not refracted.

Table I shows the results for baseline comparison, with the large baseline case shown at the top in Fig. 6. For large baselines, a significant apparent motion was observed in the EPIs, and thus refracted features yielded non-linear curves, which strongly deviated from the hyperplanes in 4D. In contrast, the non-linear characteristics of refracted feature curves were much less apparent in shorter-baseline LFs. Fig. 7 shows the horizontal and vertical EPIs for a

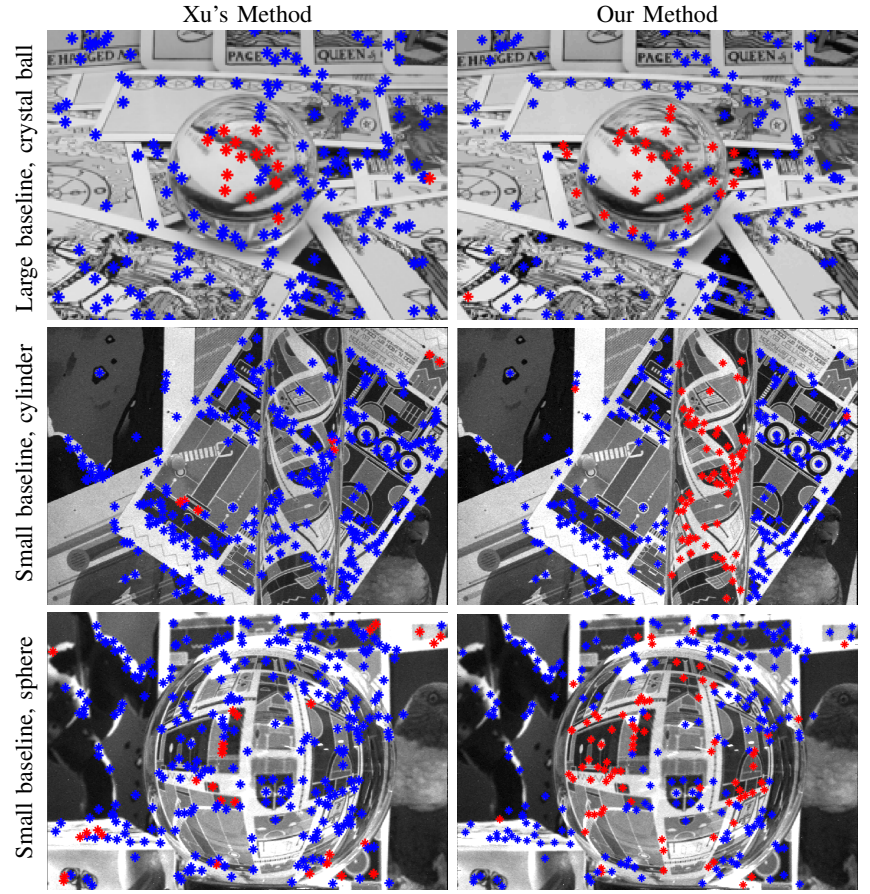

Fig. 6. Comparison of Xu's method (left), and our method (right), detecting Lambertian (blue), and refracted (red) SIFT features. In the top row, the crystal ball captured with a large baseline LF is shown (cropped) [16], where both methods detect refracted features; however, our method outperforms Xu's. In the second and third rows, a cylinder and sphere captured with a small-baseline lenslet-based LF camera. Our method successfully detects more refracted features with fewer false positives, while Xu's method does not reliably detect refracted features for small baselines.

sample refracted feature taken from the small baseline LF. The feature curves appear almost straight, despite being refracted by the crystal ball. However, we observed that the slopes were inconsistent in this particular example, which could still be used to distinguish refracted features.

Our method's TPR was 34.3\% higher Xu's method for the large baseline case, which we attributed to more accurately fitting the plane, as opposed to the single hyperplane in 4D. For the small baseline case, we attributed our 38.9\% TPR increase to accounting for slope consistency, which $\mathrm{Xu}$ did not address. Our slightly higher FPR would not be problematic as long as there are sufficiently many features. These false positives were due to occlusions, which are not yet distinguished in our implementation. However, this may still be beneficial as occlusions are non-Lambertian, and thus undesirable for most algorithms. Sampling from all the views in the LF would likely improve the results for both Xu's and our methods, as more data would improve the planar fit.

\section{Detection with the Lenslet-based LF Camera}

We investigated two different types of refractive objects, including a glass sphere and an acrylic cylinder, shown in the bottom two rows of Fig. 6. The sphere exhibited significant refraction along both the horizontal and vertical viewing axes; however, the cylinder only exhibited significant refraction perpendicular to its longitudinal axis. The refractive objects were placed within a textured scene in order to create textural details on the refractive surface. 


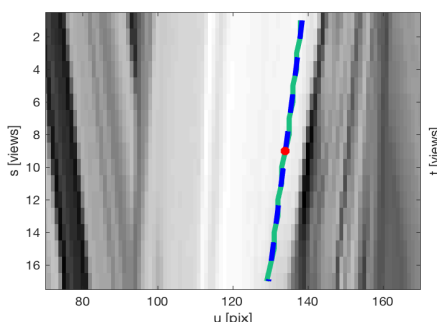

(a)

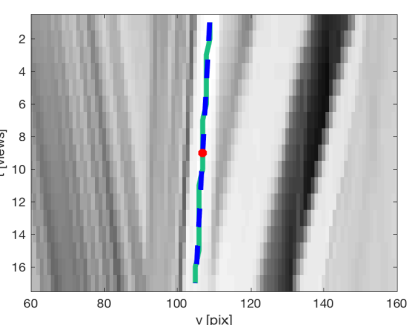

(b)
Fig. 7. Sample (a) horizontal and (b) vertical EPIs from the crystal ball LF with small baseline for refracted feature (red). Extracted feature curves (green) match the plane of best fit (dashed blue). Refracted features appear almost linear, and are thus much more difficult to detect; however, slope consistency is still an important mechanism for distinguishing refraction.

TABLE I

COMPARISON FOR BASELINES AND REFRACTIVE OBJECTS IN DISTINGUISHING REFRACTED FEATURES ${ }^{a}$

\begin{tabular}{ccccc}
\hline & \multicolumn{2}{c}{ Xu's } & \multicolumn{2}{c}{ Proposed } \\
& TPR & FPR & TPR & FPR \\
\hline Baseline & & & & \\
large $(275 \mathrm{~mm})$ & $37.1 \%$ & $\mathbf{1 . 3 \%}$ & $\mathbf{7 1 . 4 \%}$ & $3.5 \%$ \\
small $(64 \mathrm{~mm})$ & $16.6 \%$ & $\mathbf{0 \%}$ & $\mathbf{5 5 . 5 \%}$ & $3.9 \%$ \\
\hline Object & \multicolumn{3}{c}{} \\
cylinder & $12.7 \%$ & $14.8 \%$ & $\mathbf{9 0 . 9 \%}$ & $\mathbf{1 0 . 1 \%}$ \\
sphere & $27.4 \%$ & $54.2 \%$ & $\mathbf{5 3 . 4 \%}$ & $\mathbf{6 . 2 \%}$ \\
\hline
\end{tabular}

Table I shows the results of over 10 LFs taken from a variety of different viewing poses of the given refractive object. Planar error did not appear to be a strong indicator of refraction for the lenslet-based LF camera. Fig 6 shows a sample detection for the cylinder. Xu's method was unable to detect the refractive cylinder, while our method succeeded. Since the cylinder was aligned with the vertical axis, we expected non-linear behaviour for cylinder features along the horizontal axis. However, the small baseline of the camera reduced this effect, yielding EPIs similar to Fig. 7, and so the warping due to refraction through the cylinder was not apparent from this measure alone. On the other hand, slope consistency was a very strong indicator of refraction.

A refractive sphere was also investigated. A comparison of Xu's and our method is shown in Fig. 6, whereby our method successfully detected the refracted features, while Xu's failed to reliably detect the sphere. Features that were located close to the edge of the sphere appear more linear, and thus were not always detected. Other missed detections were due to specular reflections, that appeared like well-behaved Lambertian points. Finally, there are some missed detections near the middle of the sphere, where there is identical apparent motion in the horizontal and vertical hyperplanes.

\section{E. Rejecting Refracted Features for Structure from Motion}

We validated our method by examining the impact of refracted features on an SfM pipeline. We captured a sequence of LFs that gradually approached a refractive object using a lenslet-based LF camera; thus the image sequence had an increasing number of refracted features. We used Colmap, a publicly-available, modular SfM implementation [19]. The centre view of the LF was used as input to SfM. Incremental SfM was performed on an image sequence where each successive image had an increasing number of refracted features, making it increasingly difficult for SfM to converge. If SfM converged, a sparse reconstruction was produced, and the reprojection error was computed.

For each LF, SIFT features in the central view were detected, creating an unfiltered list of features, some of which were refractive. Our distinguisher was then used to remove refracted features, creating a filtered list of features. Both the unfiltered, and filtered lists of features were imported separately into the SfM pipeline, which included its own outlier rejection and bundle adjustment.

Outlier rejection schemes, such as RANSAC, are often used to reject inconsistent features, which includes refracted features. And while we observed some sequences where RANSAC successfully rejected most of the refracted features, more than $53 \%$ of inliers were actually refracted features in some cases. This suggested that in the presence of refractive objects, RANSAC is insufficient on its own for robust and accurate structure and motion estimation.

We measured the ratio of refracted features $r=i_{r} / i_{t}$, where $i_{r}$ is the number of refracted features in the image (obtained via a manually-defined mask), and $i_{t}$ is the total number of features detected in the image. We considered the reprojection error as it varied with $r$. The results are shown in Fig. 8a, in which, unsurprisingly, the error for the unfiltered case was consistently significantly higher (up to $42.4 \%$ higher for $r<0.6$ in the red case), and increased much faster than the filtered case, except when the number of inlier features became too low $(<30)$. This suggested that having a more consistent (non-refractive) feature set improves the accuracy of reconstruction. Additionally, in many cases the unfiltered case failed to converge, while the filtered case was still successful, suggesting better convergent properties. Sample scenes that prevented SfM from converging are shown in Fig. 8b, and 8c. These scenes could not be used with SfM without our refracted feature distinguisher.

However, rejecting refracted features prior SfM does not guarantee convergence for SfM, which can fail for a number of reasons. Perhaps most significantly, removing all of the refracted features reduces the number of candidate features for matching, sometimes below the threshold for minimum number of features required by Colmap to register images. In these situations, the unfiltered case is sometimes more likely to converge (albeit with much larger error) simply due to having more inlier features. This is seen in filtered case 2 in Fig. 8a. Additionally, as we move closer, the number of detected features naturally decreases, making SfM in the presence of refractive objects even more challenging.

For the cases where SfM was able to converge in the presence of refractive objects, we observed "phantom points" in the SfM reconstruction. Phantom points are points that 


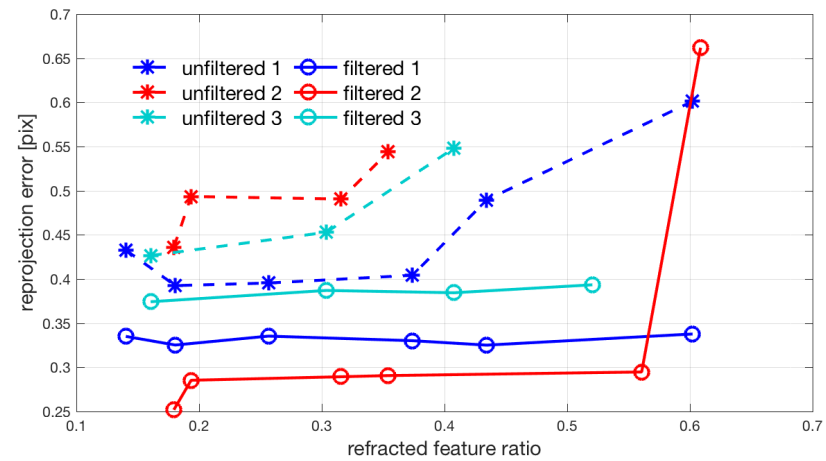

(a)

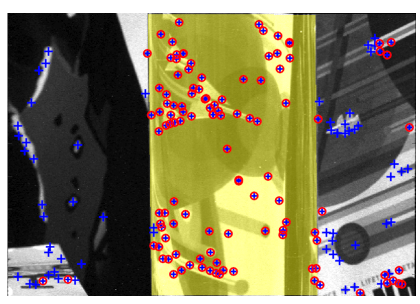

(b)

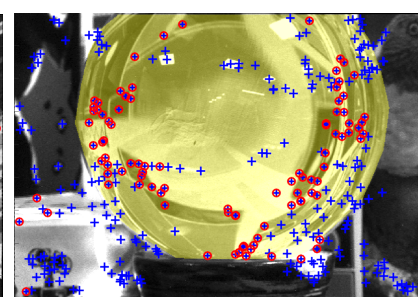

(c)
Fig. 8. (a) Rejecting refracted features with our method yielded lower reprojection errors and better convergence. SfM reprojection error vs refracted feature ratio for the unfiltered case containing refracted features (blue), and filtered case (red). The spike in error at 0.6 ratio for filtered sequence 2 is due to insufficient inliers after RANSAC. (b) and (c) show example images for the refractive cylinder and sphere (shown in yellow), respectively, that could not converge without pre-filtering for refracted features using our method. Detected features are shown in blue crosses, with features identified as refractive shown in red circles.

were placed in empty space near-but not on-the refractive object by SfM, due to refracted features counted as inliers. With our method, there were little to no such phantom points in the reconstruction. This is a subtle but important difference since the absence of information is treated very differently from incorrect information in robotics. For example, phantom points might incorrectly fill an occupancy map, preventing a robot from grasping refractive objects.

\section{CONCLUSIONS}

In this paper, we proposed a method to distinguish refracted features based on a planar fit in 4D and slope consistency. To achieve this, we extracted feature curves from the 4D LF using textural cross-correlation. For large baselines, our approach yielded higher rates of detection than previous work; however, for smaller baselines, including a lenslet-based LF camera, previous methods were unable to detect refracted objects, while our approach was successful. For these baselines, slope inconsistency proved to be a much stronger indicator of refraction than planar consistency. This is appealing for mobile robot applications, such as domestic robots that are limited in size and mass, but will have to navigate and eventually interact with refractive objects.

We also demonstrated that rejecting refracted features in monocular SfM yields lower reprojection errors, which may imply better reconstructions of the non-refractive parts of the scene in the presence of refractive objects. Further research into slope consistency, the distribution of refracted features, and LF-specific features may lead towards recovering refractive shape from features.

It is important to note that while we have developed a set of criteria for refracted features in the LF, these criteria are not necessarily limited to refracted features. Depending on the surface, specular reflections may appear as nonlinear. Poor camera calibrations may also cause Lambertian features to appear refractive in the light field. Occlusions are also occasionally detected, though they must be properly identified in future work. These types of features are typically undesirable, and so we retain features that are strongly Lambertian, and thus good candidates for matching, which ultimately leads to more robust robot performance in the presence of refractive objects.

Finally, in this paper, we explored the effect of removing the refractive content from the scene. In future work, we plan to exploit the refractive content for robot motion and refractive shape recovery.

\section{REFERENCES}

[1] C. C. Kemp, A. Edsinger, and E. Torres-Jara, "Challenges for robot manipulation in human environments," IEEE Robotics \& Automation Magazine, 2007.

[2] I. Ihrke, K. Kutulakos, H. Lensch, M. Magnor, and W. Heidrich, "Transparent and specular object reconstruction," Computer Graphics Forum, 2010.

[3] D. G. Dansereau, "Plenoptic signal processing for robust vision in field robotics," Ph.D. dissertation, University of Sydney, Jan. 2014.

[4] R. Hartley and A. Zisserman, Multiple View Geometry in Computer Vision. Cambridge, 2003.

[5] Y. mei Wei, L. Kang, B. Yang, and L. da Wu, "Applications of structure from motion: a survey," Journal of Zhejiang University-SCIENCE C (Computers \& Electronics), vol. 14, no. 7, 2013.

[6] M. Ben-Ezra and S. K. Nayar, "What does motion reveal about transparency," in ICCV, 2003.

[7] S. Wanner and B. Golduecke, "Reconstructing reflective and transparent surfaces from epipolar plane images," 35th German Conf. Pattern Recog., 2013.

[8] K. Maeno, H. Nagahara, A. Shimada, and R. Taniguchi, "Light field distortion feature for transparent object recognition," in CVPR, 2013

[9] Y. Xu, H. Nagahara, A. Shimada, and R. Taniguchi, "Transcut: Transparent object segmentation from a light-field image," CVPR, 2015.

[10] D. Tsai, D. Dansereau, T. Peynot, and P. Corke, "Image-based visual servoing with light field cameras," IEEE Robotics and Automation Letters (RAL), January 2017.

[11] I. Tosic and K. Berkner, "3d keypoint detection by light field scaledepth space analysis," in Image Processing (ICIP), 2014.

[12] J. A. Teixeira, C. Brites, F. Pereira, and J. Ascenso, "Epipolar based light field key-location detector," in Multimedia Signal Processing MMSP, Int. Workshop, 2017.

[13] O. Johannsen, A. Sulc, and B. Goldluecke, "On linear structure from motion for light field cameras," in ICCV, 2015.

[14] E. W. Weisstein, "Hyperplane," 2017. [Online]. Available: http: //mathworld.wolfram.com/Hyperplane.html

[15] R. Bolles, H. Baker, and D. Marimont, "Epipolar-plane image analysis: An approach to determining structure from motion," IJCV, 1987.

[16] M. Levoy, "The (new) stanford light field archive," 2008. [Online]. Available: http://lightfield.stanford.edu/lfs.html

[17] D. G. Lowe, "Distinctive image features from scale-invariant keypoints," IJCV, 2004.

[18] D. G. Dansereau, O. Pizarro, and S. B. Williams, "Decoding, calibration and rectification for lenselet-based plenoptic cameras," in CVPR, 2013.

[19] J. Schoenberger and J.-M. Frahm, "Structure-from-motion revisited," CVPR, 2016. 